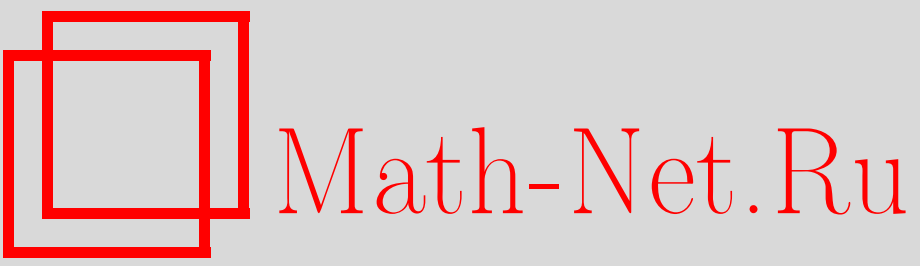

Л. Ц. Аджемян, Н. В. Антонов, А. Н. Васильев, Ренормгруппа, операторное разложение и аномальный скейлинг в простой модели турбулентной диффузии, ТМФ, 1999, том 120, номер 2, 309-314

DOI: https://doi.org/10.4213/tmf777

Использование Общероссийского математического портала Math-Net.Ru подразумевает, что вы прочитали и согласны с пользовательским соглашением

http://www. mathnet.ru/rus/agreement

Параметры загрузки:

IP : 54.162 .127 .20

26 апреля 2023 г., 15:39:13 


\section{РЕНОРМГРУППА, ОПЕРАТОРНОЕ РАЗЛОЖКНИЕ И АНОМАЛЬНЫЙ СКЕЙЛИНГ В ПРОСТОЙ МОДЕЛИ ТУРБУЛЕНТНОЙ ДИФФУЗИИ}

Аппарат ренормализационной группы и операторного разложения в модели Обухова-Крейчнана для перемешивания пассивной скалярной примеси случайным гауссовым полем скорости с коррелятором $\left\langle\mathbf{v}(t, \mathbf{x}) \mathbf{v}\left(t^{\prime}, \mathbf{x}\right)\right\rangle-\left\langle\mathbf{v}(t, \mathbf{x}) \mathbf{v}\left(t^{\prime}, \mathbf{x}^{\prime}\right)\right\rangle \propto \delta\left(t-t^{\prime}\right) \mid \mathbf{x}-$ $\left.\mathbf{x}^{\prime}\right|^{\varepsilon}$ позволяет обосновать свойство аномального скейлинга в инерционном интервале как следствие наличия в модели "опасных" составных операторов (степеней скорости локальной диссипации), чьи отрицательные критические размерности определяют значения аномальных показателей. Эти показатели вычислены во втором порядке $\varepsilon$-разложения.

В последнее время проявляется заметный интерес к изучению сравнительно простой модели турбулентного перемешивания пассивной скалярной примеси случайным гауссовым полем скорости, предложенной еше в работах Обухова [1] и Крейчнана [2] (см., например, статьи [3-18] и ссылки в них). Этот интерес связан с тем, что для структурных функций модели удается доказать наличие аномального скейлинга, свойственного, как считают, реальным турбулентным потокам, и явно вычислить соответствующие аномальные показатели, чего раньше не удавалось сделать в рамках какой-либо последовательной статистической модели. В настоящей работе на примере модели $[1,2]$ показано, что для изучения аномального скейлинга весьма эффективными оказьваются методы ренормализационной группы (РГ) и операторного разложения $(\mathrm{OP})$.

Перемешивание пассивной скалярной примеси $\theta(x)$ (везде $x \equiv(t, \mathbf{x})$ ) турбулентными пульсациями поля скорости $\mathbf{v}(x)$ описывается стохастическим уравнением

$$
\nabla_{t} \theta=\nu_{0} \Delta \theta+f, \quad \nabla_{t} \equiv \partial_{t}+v_{i} \partial_{i}
$$

где $\nu_{0}$ - коэффициент диффузии, $f(t, \mathbf{x})$ - случайная сила. Жидкость считается несжимаемой, так что поле скорости поперечно: $\partial_{i} v_{i}=0$. Статистика поля скорости предполагается гауссовой с нулевым средним и коррелятором вида

$$
\left\langle v_{i}(x) v_{j}\left(x^{\prime}\right)\right\rangle=D_{0} \frac{\delta\left(t-t^{\prime}\right)}{(2 \pi)^{d}} \int d \mathbf{k} P_{i j}(\mathbf{k})\left(k^{2}+m^{2}\right)^{-d / 2-\varepsilon / 2} \exp \left[i \mathbf{k} \cdot\left(\mathbf{x}-\mathbf{x}^{\prime}\right)\right],
$$

\footnotetext{
* Санкт-Петербургский государственный университет, Санкт-Петербург, Россия
} 
здесь $d$ - размерность пространства, $P_{i j}(\mathbf{k})=\delta_{i j}-k_{i} k_{j} / k^{2}-$ поперечный проектор, а $D_{0}>0, m>0,0<\varepsilon<2$ - параметры. Величина $1 / m$ имеет смысл интегрального масштаба турбулентности, пространственная часть выражения (2) для $\varepsilon=2 / 3$ имеет при $k \gg m$ вид колмогоровского спектра. Распределение силы $f$ также предполагается гауссовым с нулевым средним, коррелятором

$$
\left\langle f(x) f\left(x^{\prime}\right)\right\rangle=\delta\left(t-t^{\prime}\right) F(M r), \quad r \equiv\left|\mathbf{x}-\mathbf{x}^{\prime}\right|,
$$

и интегральным масштабом $L \equiv M^{-1}$. Функция $F(M r)$ считается конечной при $L \rightarrow \infty: F(0)=$ const $\neq 0$.

Структурные функции $n$-го порядка определяются как средние $S_{n}(r) \equiv\langle[\theta(x)-$ $\left.\left.\theta\left(x^{\prime}\right)\right]^{2 n}\right\rangle$, где $r \equiv\left|\mathbf{x}-\mathbf{x}^{\prime}\right|$ и $t=t^{\prime}$. Характерными длинами для этих функций являются интегральные масштабы $L, 1 / m$, а также длина диссипации $r_{d} \equiv 1 / \Lambda \equiv\left(\nu_{0} / D_{0}\right)^{1 / \varepsilon}$. Для развитой турбулентности $r_{d} \ll L, r_{d} \ll 1 / m$; таким образом, в задаче существует инерционный (конвективный) интервал $r_{d} \ll r \ll L$, поведение в котором функций типа $S_{n}(r)$ и является основным предметом исследования. Если пренебречь в (1) нелинейностью (т.е. турбулентной конвекцией), то эти функции легко вычисляются и имеют вид $S_{n}(r)=\nu_{0}^{-n} r^{2 n} F_{n}(M r)$, где $F_{n}(0)=\mathrm{const} \neq 0$. Для полной нелинейной задачи появляется зависимость от двух новых безразмерных переменных $\Lambda r$ и $m r$ :

$$
S_{n}(r)=\nu_{0}^{-n} r^{2 n} F_{n}(\Lambda r, m r, M r) .
$$

Вьполненный в [3-9] анализ показывает, что в функциях (4) можно перейти к пределу $m=0$ (что всегда предполагается в дальнейшем), тогда как их поведение при $\Lambda r \rightarrow \infty$, $M r \rightarrow 0$ (инерционный интервал) оказывается нетривиальным:

$$
S_{n}(r) \simeq C_{n} \nu_{0}^{-n} r^{2 n}(\Lambda r)^{-\alpha_{n}}(M r)^{-\beta_{n}},
$$

т.е. остается зависимость как от длины диссипации, так и от интегрального масштаба, с нелинейными по $n$ показателями $\beta_{n}$ ( "аномальный скейлинг"). Соотношения $(5)$ с явными выражениями для показателей $\alpha_{n}, \beta_{n}$ в первом порядке по $\varepsilon$ или $1 / d$ являются основным результатом работ [6-9]. В настояшей работе показано, что они легко могут быть получены методами РГ и ОР, позволяющими повысить точность вычисления показателей $\beta_{n}$ (второй порядок по $\varepsilon$ ) и применимыми к задачам более общего типа. Подробное изложение метода РГ в стохастической теории турбулентности, включая ОР и анализ составных операторов, а также подробная библиография даны в обзорной статье [19] и книге [20]. Ниже мы приводим основные утверж дения без доказательств, останавливаясь лишь на специфических свойствах модели (1)-(3).

Решение задачи разбивается на два этапа. На первом этапе методом РГ находится асимптотика функций $F_{n}$ в $(4)$ по первому аргументу $(\Lambda r \rightarrow \infty)$ при фиксированном $M r$ (считаем $m=0$ ). Это дает

$$
S_{n}(r) \simeq \nu_{0}^{-n} r^{2 n}(\Lambda r)^{-\alpha_{n}} F_{n}(M r) .
$$

Показатели степеней $\nu_{0}$ и $r$ (без учета показателя сомножителя $\Lambda r$ ) определяются каноническими размерностями рассматриваемого объекта, общая степень $r$ - его критической размерностью с обратным знаком, вид скейлинговой функции $F_{n}(M r)$ уравнением 
РГ не определяется. Формула (6) является частным случаем предсказываемого методом РГ для задачи (1)-(3) свойства инфракрасного (ИК) скейлинга. Аналогичные асимптотики могут быть получены для любых корреляционных функций; в частности, для одновременных корреляторов $S_{n k}(r)=\left\langle\Phi_{n}(x) \Phi_{k}\left(x^{\prime}\right)\right\rangle$ мономов $\Phi_{n}=\left[\partial_{i} \theta(x) \partial_{i} \theta(x)\right]^{n}$ получается

$$
S_{n k}(r) \simeq \nu_{0}^{-n-k}(\Lambda r)^{-\gamma_{n k}} F_{n k}(M r)
$$

В технике РГ показатели степеней $\alpha_{n}$ и $\gamma_{n k}$ в $(6),(7)$ вычисляются в виде рядов по параметру $\varepsilon$ из (2), который тем самым оказывается аналогом величины $\varepsilon=4-d$ в $\varepsilon$-разложении для модели $\phi^{4}$ в теории критического поведения [21, 22].

С точки зрения метода РГ в модели (1)-(3) имеется ряд упрошающих обстоятельств по сравнению с обычными моделями типа $\phi^{4}$ : положение ИК-устойчивой неподвижной точки РГ точно дается однопетлевым приближением; отсутствует ренормировка как простых полей $\theta(x)$, так и их степеней $\theta^{n}(x)$ (в квантово-полевой терминологии - “составных операторов"); параметры $M, m$ также не ренормируются. Как следствие, показатели $\alpha_{n}$ в (6) находятся точно: $\alpha_{n}=n \varepsilon$, т.е. не имеют поправок $\varepsilon^{2}, \varepsilon^{3}$ и т.д. В отличие от них показатели $\gamma_{n k}$, являюшиеся суммой $\Delta_{n}+\Delta_{k}$ критических размерностей $\Delta_{n}$ операторов $\Phi_{n}$, имеют нетривиальное $\varepsilon$-разложение. Знание этих размерностей позволяет найти асимптотику средних значений самих операторов $\Phi_{n}$ при $M / \Lambda \rightarrow 0$ :

$$
\left\langle\Phi_{n}\right\rangle \simeq C_{n} \nu_{0}^{-n}\left(\frac{M}{\Lambda}\right)^{\Delta_{n}}
$$

Соотношение (8) дает неаналитический по $M / \Lambda$ вклад в величину $\left\langle\Phi_{n}\right\rangle$; этот вклад является главным при $M / \Lambda \rightarrow 0$, если $\Delta_{n}<0$ ("опасные операторы" - в терминологии [23]), как это и оказывается в действительности: в первом порядке по $\varepsilon$ получается $\Delta_{n}=-2 n(n-1) \varepsilon /(d+2)($ cp. $[8,9])$. Строго говоря, из-за смешивания при ренормировке моном $\Phi_{n}$ является конечной линейной комбинацией “базисных операторов" с определенными размерностями $\Delta_{k}, k \leqslant n$; в представлениях (7) и (8) оставлен только главный вклад с максимальным $\left|\Delta_{k}\right|$.

Скейлинговые функции $F_{n}(M r), F_{n k}(M r)$ в соотношениях типа (6), (7) могут быть вычислены в форме рядов по $\varepsilon$, но такое представление непригодно на втором этапе решения задачи - этапе исследования их асимптотики при $M r \rightarrow 0$, поскольку реальным параметром разложения тогда оказывается $\varepsilon \ln (M r)$. Суммирование таких "больших ИК-логарифмов", в отличие от “больших УФ-логарифмов" типа $\ln (\Lambda r)$, выходит за рамки метода РГ, так как вид скейлинговых функций самим уравнением РГ никак не определяется. Как и в теории критического поведения [21, 22], асимптотика $M r \rightarrow 0$ может быть найдена в рамках обшего решения уравнений РГ с помошью техники ОР. В этом 
случае произведение двух (ренормированных) составных операторов $O_{1}\left(x_{1}\right) O_{2}\left(x_{2}\right)$ (например, $\theta^{n}$ или ренормированных аналогов мономов $\left.\Phi_{n}\right)$ при $t_{1}=t_{2}=t, \mathbf{r} \equiv \mathbf{x}_{1}-$ $\mathbf{x}_{2} \rightarrow 0$ и фиксированном "центре тяжести" $\mathbf{x} \equiv\left(\mathbf{x}_{1}+\mathbf{x}_{2}\right) / 2=$ const представляется в виде

$$
O_{1}\left(x_{1}\right) O_{2}\left(x_{2}\right)=\sum_{\alpha} C_{\alpha}(\mathbf{r}) O_{\alpha}(\mathbf{x}, t)
$$

где $C_{\alpha}$ - аналитические по $(M r)^{2}$ коэффициенты, а суммирование в обшем случае идет по всем локальным составным операторам $O_{\alpha}(x)$ с определенными критическими размерностями $\Delta_{\alpha}$. Корреляторы типа (6), (7) получаются усреднением соотношений (9), при этом в правых частях возникают средние $\left\langle O_{\alpha}(x)\right\rangle \propto M^{\Delta_{\alpha}}$, которым соответствуют вклады вида $(M r)^{\Delta_{\alpha}}$ в скейлинговых функциях $F_{n}(M r), F_{n k}(M r)$. Ведущие члены асимптотики $M r \rightarrow 0$ определяются операторами с наименьшим значением $\Delta_{\alpha}$, в обычных моделях типа $\phi^{4}$ главный член порождается простейшим оператором $O_{\alpha}(x)=1 \mathrm{c}$ $\Delta_{\alpha}=0$. Однако в модели (1)-(3) критические размерности всех операторов $\Phi_{n}$ отрицательны (см. выше), и поэтому именно они дают главный вклад в асимптотику $M r \rightarrow 0$. Операторы, содержащие поля $\theta$ без производной $\partial$, критические размерности которых также отрищательны, не дают вклада в ОР величин типа (6), (7) в силу инвариантности последних относительно сдвига $\theta \rightarrow \theta+$ const поля $\theta(x)$.

Если бы в ОР каждой функции $(6),(7)$ давали вклад все операторы $\Phi_{n}$, для нахождения асимптотики $M r \rightarrow 0$ эти вклады нужно было бы как-то суммировать, поскольку спектр их размерностей не ограничен снизу (нет "самого опасного"). Именно так обстоит дело в более реалистических моделях развитой турбулентности (см. $[19,20,23])$. В модели (1)-(3) такой проблемы не возникает: анализ диаграмм теории возмущений показывает, что число полей $\theta$ в любом составном операторе $O_{\alpha}(x)$, входящем в правую часть соотношения (9), не может превосходить полного числа полей в его левой части. Таким образом, для величин (6), (7) получаем выражения

$$
\begin{aligned}
S_{n}(r) & \simeq \nu_{0}^{-n} r^{2 n}(\Lambda r)^{-n \varepsilon} \sum_{s \leqslant n} C_{n, s}(M r)^{\Delta_{s}}, \\
S_{n k}(r) & \simeq \nu_{0}^{-n-k}(\Lambda r)^{-\Delta_{n}-\Delta_{k}} \sum_{s \leqslant n+k} C_{n k, s}(M r)^{\Delta_{s}}
\end{aligned}
$$

с некоторыми зависящими от $\varepsilon$ и $d$ коэффициентами $C_{n, s}, C_{n k, s}$ и с возможными поправками порядка $(M r)^{2+O(\varepsilon)}$. Поскольку размерности $\Delta_{s}$ отрицательны и монотонно возрастают по модулю с ростом $s$, главным при $M r \rightarrow 0$ в (10), (11) является слагаемое с максимальным $s$, т.е. $s=n$ в (10) и $s=n+k$ в (11):

$$
\begin{aligned}
S_{n}(r) & \simeq C_{n} \nu_{0}^{-n} r^{2 n}(\Lambda r)^{-n \varepsilon}(M r)^{\Delta_{n}} \\
S_{n k}(r) & \simeq C_{n k} \nu_{0}^{-n-k}(\Lambda r)^{-\Delta_{n}-\Delta_{k}}(M r)^{\Delta_{n+k}} .
\end{aligned}
$$

Мы вычислили критические размерности $\Delta_{n}$ с точностью до членов $\varepsilon^{2}$ включительно, что потребовало расчета соответствуюших констант ренормировки в двухпетлевом 
приближении. Результат имеет вид

$$
\begin{aligned}
\Delta_{n}= & -\frac{2 n(n-1) \varepsilon}{d+2}+\frac{\varepsilon^{2} n(n-1)}{(d-1)(d+2)^{3}(d+4)^{2}} \times \\
& \times\left[-4(d+1)(d+4)^{2}+3(d-1)(d+2)(d+4)(d+2 n) h(d)-\right. \\
& -4(d+1)(d+2)(d+3 n-2) h(d+2)]+O\left(\varepsilon^{3}\right),
\end{aligned}
$$

где обозначено

$$
h(d) \equiv F\left(1,1 ; \frac{d}{2}+2 ; \frac{1}{4}\right)=\sum_{k=0}^{\infty} \frac{k ! \Gamma\left(\frac{d}{2}+2\right)}{4^{k} \Gamma\left(\frac{d}{2}+2+k\right)}
$$

и $F(a, b ; c ; z)$ - гипергеометрический ряд (см., например, [24]). Для целых $d$ ряд в (15) суммируется явно, что, в частности, дает $h(2)=1-24 \ln (4 / 3), h(3)=10(\pi \sqrt{3}-16 / 3)$.

Уравнения Швингера позволяют доказать точное равенство $\Delta_{1}=0$, что согласуется с полученным в [2] точным решением для функции $S_{1}(r)$. Асимптотики $(12),(13)$ совпадают с результатом, полученным другим способом в работах $[8,9]$, где показатели $\Delta_{n}$ были вычислены в первом порядке по $\varepsilon$. Они согласуются также с результатами, полученными в $[6,7]$ в первом порядке по $1 / d$.

Заметим, что набор операторов $\Phi_{n}$ замкнут относительно "слияния" в том смысле, что главный вклад в ОР для коррелятора $\left\langle\Phi_{n} \Phi_{m}\right\rangle$ дается оператором из того же семейства $\Phi_{n+m}$ c суммарным индексом $n+m$. Этот факт вместе с вытекаюшим из явного вида $\Delta_{n}$ неравенством $\Delta_{n}+\Delta_{m}>\Delta_{n+m}$ означает, что корреляторы степеней локальной диссипации в модели (1)-(3) демонстрируют мультифрактальное поведение (ср. $[25,26])$. Заметим также, что термин "аномальный скейлинг" обычно связывают с нелинейностью по $n$ показателей $\beta_{n}$ в соотношениях типа (5), хотя исключением является именно случай линейных показателей: например, в соотношениях (13) все показатели нелинейны.

Изложенный выше подход обобшается на случаи наличия сжимаемости, анизотропии, конечного времени корреляции, а также на случай корреляционных функций различных тензорных операторов (см. [27-29]).

В заключение упомянем кратко основанный на РГ и ОР подход к более реалистической модели развитой турбулентности - стохастическому уравнению Навье-Стокса с внешней случайной силой (подробнее см. обзор [19] и книгу [20]). "Опасные операторы" в этой задаче возникают лишь при конечных значениях РГ-параметра $\varepsilon$, и достоверно идентифицировать их можно только тогда, когда их размерности найдены точно с помощью уравнений Швингера или тождеств Уорда. Они входят в ОР в виде бесконечных семейств с не ограниченным снизу спектром размерностей, и для нахождения асимптотик $M r \rightarrow 0$ их вклады в ОР необходимо дополнительно суммировать. Такое суммирование, вьполненное в [23] для разновременных корреляторов, обнаружило их сингулярную зависимость от $M$, что феноменологически объясняется известным эффектом переноса турбулентных вихрей как целого крупномасштабными движениями ("sweeping effects") (см. [30]). Это показывает, что данный эффект "не теряется" при использовании метода РГ, как иногда считают [31, 32], если аппарат РГ используется в сочетании с ОР и вне рамок $\varepsilon$-разложения. Анализ зависимости от $M$ одновременных галилеево-инвариантных величин типа структурных функций (4) для стохастического 
уравнения Навье-Стокса требует явного нахождения критических размерностей всех инвариантных скалярных "опасных операторов" и суммирования их вкладов в ОР типа (9). Очевидно, что это гораздо более сложная проблема по сравнению с изучением ИК-асимптотик упрошенной модели (1)-(3).

Благодарности. Работа выполнена при поддержке Российского фонда фундаментальных исследований (грант № 99-02-16783) и Конкурсного центра фундаментального естествознания Госкомвуза (грант № 97-0-14.1-30).

\section{Список литературы}

[1] А. М. Обухов. Изв. АН СССР. Сер. геофиз. 1949. Т. 13. С. 58.

[2] R. H. Kraichnan. Phys. Fluids. 1968. V. 11. P. 945.

[3] R. H. Kraichnan. Phys. Rev. Lett. 1994. V. 72. P. 1016; 1997. V. 78. P. 4922.

[4] R. H. Kraichnan, V. Yakhot, S. Chen. Phys. Rev. Lett. 1995. V. 75. P. 240.

[5] V. Yakhot. Phys. Rev. E. 1997. V. 55. P. 329.

[6] M. Chertkov, G. Falkovich, I. Kolokolov, V. Lebedev. Phys. Rev. E. 1995. V. 52. P. 4924.

[7] M. Chertkov, G. Falkovich. Phys. Rev. Lett. 1996. V. 76. P. 2706.

[8] K. Gawȩdzki, A. Kupiainen. Phys. Rev. Lett. 1995. V. 75. P. 3834

[9] D. Bernard, K. Gawȩdzki, A. Kupiainen. Phys. Rev. E. 1996. V. 54. P. 2564.

[10] D. Gutman, E. Balkovsky. Phys. Rev. E. 1996. V. 54. P. 4435.

[11] A. Pumir. Europhys. Lett. 1996. V. 34. P. 25; 1997. V. 37. P. 529; Phys. Rev. E. 1998. V. 57. P. 2914.

[12] G. L. Eyink. Phys. Rev. E. 1996. V. 54. P. 1497; G. L. Eyink, J. Xin. Phys. Rev. Lett. 1996. V. 77. P. 4166.

[13] A. Pumir, B. I. Shraiman, E. D. Siggia. Phys. Rev. E. 1997. V. 55. P. R1263.

[14] E. Balkovsky, G. Falkovich, V. Lebedev. Phys. Rev. E. 1997. V. 55. P. R4881.

[15] M. Chertkov. Phys. Rev. E. 1997. V. 55. P. 2722.

[16] A. L. Fairhall, B. Galanti, V.S. L'vov, I. Procaccia. Phys. Rev. Lett. 1997. V. 79. P. 4166.

[17] O. Gat, V. S. L'vov, I. Procaccia. Phys. Rev. E. 1997. V. 56. P. 406.

[18] U. Frisch, A. Mazzino, M. Vergassola. Phys. Rev. Lett. 1998. V. 80. P. 5532.

[19] Л. Ц. Аджемян, Н. В. Антонов, А. Н. Васильев. УФН. 1996. Т. 166. С. 1257.

[20] L. Ts. Adzhemyan, N. V. Antonov, A. N. Vasiliev. Field Theoretic Renormalization Group in Fully Developed Turbulence. London: Gordon and Breach Publ., 1999.

[21] J. Zinn-Justin. Quantum Field Theory and Critical Phenomena. Oxford: Clarendon, 1989.

[22] А. Н. Васильев. Квантово-полевая ренормгруппа в теории критического поведения и стохастической динамике. С.-П.: Изд-во ПИЯФ, 1998.

[23] Л. Ц. Аджсемян, Н. В. Антонов, А. Н. Васильев. ЖЭТФ. 1989. Т. 95. С. 1272; Н. В. Антонов. Зап. научн. семин. ЛОМИ. 1988. Т. 169. С. 18.

[24] А. П. Прудников, Ю.А. Брычков, О. И. Маричев. Интегралы и ряды. Специальные функции. М.: Наука, 1983.

[25] B. Duplantier, A. Ludwig. Phys. Rev. Lett. 1991. V. 66. P. 247.

[26] G. L. Eyink. Phys. Lett. A. 1993. V. 172. P. 355.

[27] L. Ts. Adzhemyan, N. V. Antonov, A. N. Vasil'ev. Phys. Rev. E. 1998. V. 58. P. 1823.

[28] L. Ts. Adzhemyan, N. V. Antonov. Phys. Rev. E. 1998. V. 58. P. 7381.

[29] N. V. Antonov. Anomalous scaling regimes of a passive scalar advected by the synthetic velocity field. Preprint SPBU IP-98-16. St-P.: St-Petersburg Univ., 1998; chao-dyn/9808011.

[30] R. H. Kraichnan. Phys. Fluids. 1964. V. 7. P. 1723; 1965. V. 8. P. 575.

[31] S. Chen, R. H. Kraichnan. Phys. Fluids A. 1989. V. 1. P. 2019.

[32] S. L. Woodruff. Phys. Fluids. [Formely Phys. Fluids A]. 1994. V. 6. P. 3051. 